\title{
High-Throughput Non-Orthogonal Interleaved Random Space-Time Coding for Multi-Source Cooperation
}

\author{
Rong Zhang and Lajos Hanzo \\ School of ECS., Univ. of Southampton, SO17 1BJ, UK. \\ Tel: +44-23-80-593 125, Fax: +44-23-80-593 045 \\ Email: 1h@ecs.soton.ac.uk, http://www-mobile.ecs.soton.ac.uk
}

\begin{abstract}
In this paper, we propose a novel distributed Interleaved Random Space-Time Code (IR-STC) designed for MultiSource Cooperation (MSC) employing various relaying techniques, namely Amplify-Forward, Decode-Forward, Soft-Decode-Forward and Differential-Decode-Forward. We characterise the achievable slot utilisation efficiency and introduce a two-phase communication regime for our IR-STC aided MSC. A matrix based formalism is used for describing our IR-STC scheme and a novel Structured Embedded (SE) random interleaver generation method is proposed. Furthermore, the Bit Error Ratio (BER) performance of our IR-STC is characterised in conjunction with various relaying techniques under different inter-source Nakagamim fading channels.
\end{abstract}

\section{INTRODUCTION}

Cooperative diversity [1] relying on a distributed (virtual) Multiple Input Multiple Output (MIMO) system is capable of eliminating the correlated fading induced diversity-gain erosion of co-located MIMO elements imposed by the omni-present shadow fading. Hence this novel technique is capable of improving the Bit Error Ratio (BER) performance, while supporting a high throughput as well as providing an improved cell-edge coverage [2]. It has the potential of beneficially combining the traditional infrastructure based wireless networks and the ad-hoc wireless network philosophy [3]. Most recent research in the literature was dedicated to the aspects of information-theoretic analysis, to creating practical relaying techniques and to the investigation of distributed Space-Time Code (STC) designs [4]-[6].

Recently, the Cooperative Multiple Access (CMA) channel has attracted substantial research interests [6], where multiple sources forming a cluster of cooperating nodes communicate with the destination, which is also known as Multi-Source Cooperation (MSC) [7], [8]. Inspired by the multilayer Interleave-Division-Multiplexing (IDM) aided STC concept [9], an error-resilient yet high-throughput Interleaved Random STC (IR-STC) scheme was contrived for MSC in [10]. The achievable rate, power-efficiency and the network architecture's flexibility were analyzed and were shown to be superior to the corresponding features of the conventional distributed Orthogonal Space Time Block Codes (OSTBC) [11].

Our IR-STC designed for MSC in [10] exhibits several beneficial properties. Explicitly, it achieves a high-throughput as a benefit of its high slot utilisation efficiency with the aid of the superposition coding concept [12], [13]. Furthermore, it has a low BER thanks to the powerful iterative receiver employed. Finally, our IR-STC design constitutes a non-orthogonal scheme, which is capable of approaching the (cooperative) multiple access channel's capacity [6]. Additionally, the IR-STC proposed has the benefit of operating with the aid of using autonomously generated random interleavers,

Acknowledgments: The work reported in this paper has formed part of the Core 4 Research Programme of the Virtual Center of Excellence in Mobile and Personal Communications, Mobile VCE, www.mobilevce.com, whose funding support, including that of EPSRC, is gratefully acknowledged. Fully detailed technical reports on this research are available to Industrial Members of Mobile VCE. which facilitates their cooperation without any central controller, even without knowing the number of nodes ${ }^{1}$. Given the above-mentioned attributes, we refer to the proposed scheme as a decentralized nonorthogonal IR-STC.

In short, our goal is to quantify the slot utilisation efficiency achieved by MSC compared to conventional Single Source Cooperation (SSC) and propose a novel Structured Embedded (SE) interleaver design method emerging from the analysis of our IR-STC concept. Furthermore, we investigate several relaying techniques in the context of our IR-STC aided MSC, such as the Amplify-Forward (AF), Decode-Forward (DF), Soft-Decode-Forward (SDF) and DifferentialDecode-Forward (DDF) technique. Against this background, the novel contribution of this paper is that we design a decentralized high-throughput non-orthogonal IR-STC scheme suitable for MSC and characterize its achievable performance, when employing various relaying techniques.

The rest of the paper is organized as follows. In Section II, we describe the MSC scenarios considered, highlight its slot utilisation efficiency and introduce our novel IR-STC architecture designed for MSC. In Section III, we analyse our IR-STC and propose an efficient SE interleaver generation method. In Section IV, we investigate the performance of the IR-STC scheme employing different relaying techniques using simulations. Finally, we conclude our discourse in Section V.

Notation: Throughout the paper, lower (upper) case boldface letters will represent column vectors (matrices). The all-one vector is denoted as $\mathbf{1}_{N}=[1, \ldots, 1]^{T}$. The superscripts $(\cdot)^{T}$ and $(\cdot)^{*}$ denote transposition and complex conjugation, respectively. The superscript $(\cdot)^{(1)}$ and $(\cdot)^{(2)}$ denotes Phase-I cooperation and PhaseII cooperation. The subscript $[\cdot]_{(k)}$ denotes the exclusion of the $k$ th element. The subscript $\mathbf{A}_{(k,:)}$ stands for the $k$ th row of matrix $\mathbf{A}$.

\section{IR-STC AIDED Multi-Source CoOperation}

\section{A. Assumptions}

Consider a cluster of single-antenna sources cooperatively communicating with a destination employing a single receive antenna resulting in a Virtual Multiple Input Single Output (VMISO) system. In this VMISO cluster, we assume having a total of $N$ Cooperating Sources (CS), $K$ Active Sources (AS) and $(N-K)$ Relaying Sources (RS).

Cooperative communications typically entail two phases. In PhaseI cooperation, the source information emanating from all $K$ ASs is broadcast to all $N$ CSs in a Time Division Duplex (TDD) manner under the assumption of perfect synchronization. By contrast, PhaseII cooperation is defined as the joint transmission of a combined IR-STC signal by the concerted action of all the $N$ CSs.

\footnotetext{
${ }^{1}$ This is in contrast to the conventional distributed OSTBC, where each node emulates a specific virtual array element of a structured STC. Thus, our design avoids the requirement of a centralised code allocation procedure.
} 


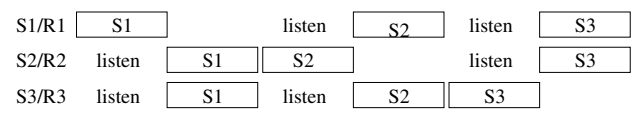

Single Source Cooperation

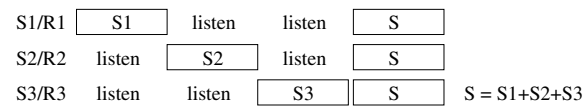

Multi Source Cooperation
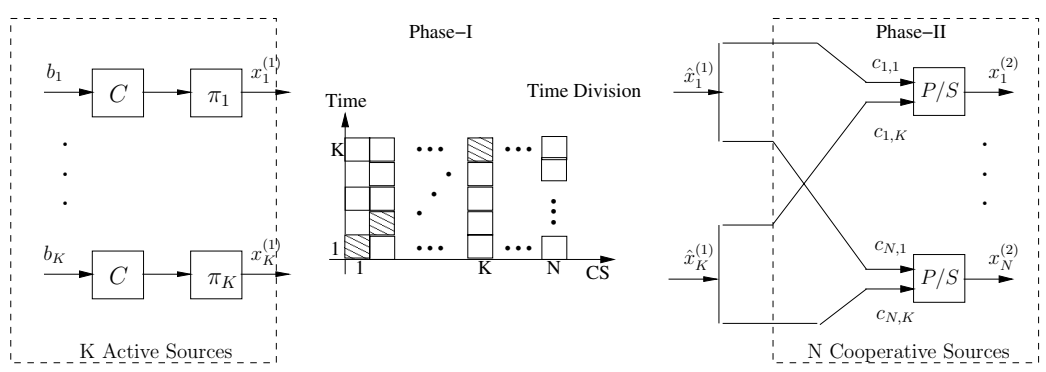

Fig. 1. (a) Slot utilisation efficiency of Multi-Source Cooperation and Single Source Cooperation.; (b) Block diagram of Interleaved Random Space-Time Code aided Multi-Source Cooperation

We assume that all inter-source channels denoted as $h_{s, s}$ and the source-destination channel denoted as $h_{s, d}$ experience independent identically distributed (i.i.d) Nakagami-m fading obeying the Probability Density Function (PDF) of [14]:

$$
p_{Z}(x)=\frac{2 m^{m}}{\Gamma(m) \Omega^{m}} x^{2 m-1} \exp \left\{-\frac{m x^{2}}{\Omega}\right\}, x \geq 0
$$

where $m \geq 0.5$ is the Nakagami-m fading parameter, $\Omega=\mathrm{E}\left\{x^{2}\right\}$ is the variance of $x$ and $\Gamma(\cdot)$ is the Gamma function. In this paper, the inter-source channel $h_{s, s}$ is assumed to be asymmetric, i.e. we have $h_{k, n} \neq h_{n, k}$, where $h_{k, n}$ represents the inter-source channel between source $k$ and source $n$, which tend to be in close proximity of each other $^{2}$. We also assume that the inter-source channels benefit from a higher effective Signal-to-Noise-Ratio (SNR), i.e. we have $\gamma_{s, s}>$ $\gamma_{s, d}$. Furthermore, $m>1$ is used only for the inter-source channels, where an SNR-based node pre-selection scheme may be used for spotting the specific CSs benefiting from a high-quality channel.

\section{B. Slot utilisation efficiency}

As seen in Fig. 1(a), where $N=K=3$, in conventional $\mathrm{SSC}$, each source broadcasts its information to all $(N-1) \mathrm{CSs}$ during the Phase-I cooperation, which is followed by a joint relaying of their information to the destination by the concerted action of the $(N-1)$ CSs in Phase-II cooperation. An entire cooperative transmission phase is concluded, when all $K$ ASs completed their cooperation. By contrast, MSC is constituted by a full cycle of information broadcasting from all $K$ ASs to all $N$ CSs during Phase-I cooperation, followed by their joint transmission to the destination during Phase-II cooperation, where each CS transmits all $K$ ASs' information. Therefore, each CS simultaneously transmits multiple sources' information with the aid of their superposition, resulting in a high throughput. This implies that each source is served simultaneously by multiple CSs, which are chosen to be those that experience a high-quality inter-source channel and hence the entire set of ASs benefits from a high diversity gain.

Let us define the slot utilisation efficiency of a cooperative scheme $\eta_{s}$ as the ratio between the time duration required for transmitting all $K$ ASs' information in a non-cooperative manner and that necessitated in a cooperative manner. Assume that the information broadcast phase of each source requires a time duration of $T_{1}$, while the joint transmission in Phase-II takes a time duration of $T_{2}$. Thus the slot utilisation efficiency of SSC is $\eta_{s-s s c}=T /\left(K T_{1}+K T_{2}\right)$, while that of MSC is $\eta_{s-m s c}=T /\left(K T_{1}+T_{2}\right)$, where $T$ is the time duration required for transmitting all $K$ ASs' information in a non-cooperative manner, which is $T=K T_{1}$ when TDMA is used. Therefore, MSC is preferable to SSC in terms of its higher slot utilisation efficiency.

${ }^{2}$ Since each of the $K$ ASs transmits its information in a Phase-I cooperation slot, it is reasonable to assume that the fading envelope is constant in that slot and fades independently for the different slots.

\section{IR-STC Construction}

1) Phase-I cooperation: As seen in Fig. 1(b), we assume that each BPSK modulated AS employs a repetition code $\mathrm{C}$ of rate $r \leq 1 / N$ and a source-specific interleaver $\pi_{k}$. During Phase-I cooperation, the $k$ th AS transmits a repetition coded and randomly interleaved bitstream $x_{k}^{(1)}=\pi_{k}\left[\mathrm{C}\left(b_{k}\right)\right], k \in[1, K]$. Then, depending on whether the inter-source channel $h_{s, s}$ is known at all CSs, two different transmission modes can be employed, namely coherent modulation and non-coherent modulation.

Coherent modulation: During the $k$ th of the $K$ number of available TDD time-slots, the $n$th CS receives the signal transmitted from the $k$ th AS, yielding the received signal $y_{k, n}^{(1)}=h_{k, n} x_{k}^{(1)}+$ $n_{0}, k \in[1, K], n \in[1, N]_{(k)}$, where $n_{0} \sim \mathcal{C N}\left(0, N_{0}\right)$ denotes the complex-valued Additive White Gaussian Noise (AWGN). In this scenario, three relaying techniques are considered.

- Amplify Forward. The signal $y_{k, n}^{(1)}$ received by the $n$th CS is scaled to meet the average power constraint, yielding:

$$
\hat{x}_{k, n}^{(1)}=\frac{y_{k, n}^{(1)}}{\sqrt{N_{0}+\left|h_{k, n}\right|^{2}}} .
$$

- Soft Decode Forward. The soft value $(\mathcal{L})$ of the signal $y_{k, n}^{(1)}$ received at the $n$th CS is calculated as [15] $\mathcal{L}=4\left(\left|h_{k, n}\right|^{2} x_{k}^{(1)}+\right.$ $\left.\mathcal{R}\left\{h_{k, n}^{*} n\right\}\right) / N_{0}$. This is then scaled to meet the average power constraint:

$$
\hat{x}_{k, n}^{(1)}=\frac{\mathcal{L} N_{0}}{4\left|h_{k, n}\right| \sqrt{N_{0}+\left|h_{k, n}\right|^{2}}} .
$$

Eq (3) essentially describes an AF technique in an uncoded or repetition coded system, because the soft value $\mathcal{L}$ can be viewed as an equivalent analogue-valued received signal.

- Decode Forward. The signal $y_{k, n}^{(1)}$ received by the $n$th CS is subject to BPSK hard detection, resulting in

$$
\hat{x}_{k, n}^{(1)}=\operatorname{sign}\left(\mathcal{R}\left\{h_{k, n}^{*} y_{k, n}^{(1)}\right\}\right) .
$$

Non-coherent modulation: When $h_{s, s}$ is unknown at the CSs, non-coherently detected differentially encoded BPSK (DBPSK) modulation can be employed. Then the transmitted bit-stream is expressed as $s_{k}^{(1)}(i)=s_{k}^{(1)}(i-1) x_{k}^{(1)}(i), i \in[1, M]$, where $M$ is the length of bit-stream $x_{k}^{(1)}$ and $s_{k}^{(1)}(0)=1$ is a dummy bit used by the DBPSK detector as a reference. Thus, we have $y_{k, n}^{(1)}=h_{k, n} s_{k}^{(1)}+n_{0}$.

Let us assume the presence of slow fading. Then $h_{s, s}$ may be considered to be constant over two consecutive bits, hence noncoherent detection is performed according to:

$$
\begin{aligned}
\hat{x}_{k, n}^{(1)}(i) & =\operatorname{sign}\left(\mathcal{R}\left\{y_{k, n}^{(1) *}(i-1) y_{k, n}^{(1)}(i)\right\}\right) \\
& =\operatorname{sign}\left(\mathcal{R}\left\{\left|h_{k, n}\right|^{2} x_{k}^{(1)}(i)+v\right\}\right),
\end{aligned}
$$

where $v \sim \mathcal{C N}\left(0,2\left|h_{k, n}\right|^{2} N_{0}\right)$ is a complex-valued AWGN component having a doubled noise variance in comparison to coherent detection, where the latter relies on accurate channel knowledge. 
When comparing these four relaying techniques, Eq. (2) and Eq. (3) retain the original signal, but scale both the signal and the noise component, while Eq. (4) and Eq. (5) assume first detecting and then reconstructing the signal, depending on the channel quality. We refer to the first two techniques as non-regenerative relay techniques and to the latter two as regenerative relay techniques.

2) Phase-II cooperation: Following Phase-I cooperation, each of the $N$ CSs detects/scales all the $K$ ASs' bit-streams according to the the above four relaying techniques characterized by Eq. (2)-Eq. (5). When considering the $n$th of the $N$ CSs, the joint IR-STC codeword is constructed as follows

- Codewords generation: The $n$th CS forms $K$ parallel streams $c_{n, k}(i)=\hat{x}_{k, n}^{(1)}[N(i-1)+n], i \in[1, M / N], k \in[1, K]$. These $K$ streams are Parallel-to-Serial (P/S) converted to $c_{n}$.

- Multilayer mapping: Then the signal transmitted from the $n$th CS becomes $x_{n}^{(2)}(i)=\left(1 / \sqrt{L_{n}}\right) \sum_{l=1}^{L_{n}} e^{j \theta_{n, l}} c_{n}\left[L_{n}(i-1)+\right.$ $l], i \in\left[1, M K / N L_{n}\right]$, where $L_{n}$ is referred to as the number of layers contributed by the $n$th CS, while $\theta_{n, l} \in[0, \pi)$ denotes the layer-specific phase rotation. In this treatise, we assume $L_{n}=L$ and $\theta_{n, l}=\theta_{l}, \forall n \in[1, N]$.

3) Iterative receiver: An iterative receiver is employed at the destination of Phase-II cooperation, where either optimum but complex Maximum-Likelihood (ML) detection or reduced-complexity suboptimum Interference Cancellation (IC) may be employed [9]. Employing different relaying techniques requires different amount of inter-source channel knowledge at the destination. For the regenerative relay techniques of Eq. (4) and Eq. (5), no inter-source channel knowledge is required at the destination, while for the non-regenerative relay techniques of Eq. (2) and Eq. (3), inter-source channel knowledge is required at the destination. However for SDF, the knowledge of the inter-source channel's magnitude $\left|h_{s, s}\right|$ at the destination is sufficient.

\section{SE IR-STC DESIGN}

\section{A. Matrix based IR-STC analysis}

Our IR-STC scheme employs a random distributed ${ }^{3}$ source-specific interleaver for differentiating the various sources. Here we adopt a matrix representation for the $k$ th AS's IR-STC. In our scheme, the information source signal $\mathbf{b}_{k}$ of length $P$ is firstly repetition coded and then randomly interleaved, yielding the sequence $\mathbf{x}_{k}^{(1)}$ of length $M=P / r$, obeying $\mathbf{x}_{k}=\pi_{k}(\mathbf{R}) \mathbf{b}_{k}$, where:

$$
\mathbf{R}=\operatorname{diag}\left[\mathbf{1}_{1 / r}, \ldots, \mathbf{1}_{1 / r}\right]_{(M \times P)}
$$

is the repetition code's matrix. The random source-specific interleaver $\pi_{k}$ of Fig. 1 permutes the corresponding rows in the matrix $\mathbf{R}$, yielding the matrix $\mathbf{G}=\pi_{k}(\mathbf{R})$. The $\mathrm{STC}$ matrix $\mathbf{C}_{k}$ of size $(M / N \times N)$ is constructed according to $\mathbf{C}_{k}=\boldsymbol{\Theta}_{k} \mathbf{B}_{k}$, where we have

$$
\begin{aligned}
\boldsymbol{\Theta}_{k} & =\left[\begin{array}{ccc}
\mathbf{G}_{(1,:)} & \cdots & \mathbf{G}_{(N,:)} \\
\vdots & \ddots & \vdots \\
\mathbf{G}_{(M-N+1,:)} & \cdots & \mathbf{G}_{(M,:)}
\end{array}\right]_{(M / N \times P N)} \\
\mathbf{B}_{k} & =\operatorname{diag}\left[\mathbf{b}_{k}, \ldots, \mathbf{b}_{k}\right]_{(P N \times N)} .
\end{aligned}
$$

Our IR-STC employs the random distributed source-specific interleaver $\pi_{k}$, which results in a distinct source-specific matrix $\boldsymbol{\Theta}_{k}$. Then these $K$ distinct IR-STCs are superimposed and transmitted simultaneously for the sake of supporting a high throughput. This random construction is different from the one proposed in [16], where

\footnotetext{
${ }^{3}$ The 'distributed' nature of the source-specific random interleaver emphasizes the fact that in contrast to the centrally controlled random interleaver assignment regime of classic cellular IDMA-style systems [9], here the interleavers are assigned autonomously by the ASs.
}

\begin{tabular}{|l||l|c|c|c|}
\hline Info. bit length & 1024 \\
Modulation Type & $\mathrm{BPSK}$ & \multicolumn{4}{l|}{} \\
Code-rate & $\mathrm{r}=1 / 8$ \\
Cluster Info. & $\mathrm{N}=4, \mathrm{~K}=4$ & & \\
Receiver & $\mathrm{IC}, 30$ iterations \\
\hline \hline & $\mathrm{AF}$ & $\mathrm{SDF}$ & $\mathrm{DF}$ & $\mathrm{DDF}$ \\
\hline Cooperating Source & $\left|h_{s, s}\right|$ & $h_{s, s}$ & $h_{s, s}$ & - \\
Destination & $h_{s, s}$ & $\left|h_{s, s}\right|$ & - & - \\
\hline
\end{tabular}

TABLE I

SUMMARY OF THE SIMULATION PARAMETERS.

each CS transmits a random linear combination of the columns of an existing OSTBC. Let us denote the code-rate of our IR-STC by $r_{I R-S T C}=r N$. When ignoring the throughput reduction imposed by Phase-I cooperation for the sake of a simple argument, the effective throughput of the cluster employing our multilayer IR-STC may be expressed as:

$$
\eta_{I R-S T C}=r_{I R-S T C} \times L .
$$

For example, when $r=1 / 8$-rate repetition coded $N=K=4$ sources are in a cluster and $L=7$ layers are superimposed at each CSs, an aggregate rate as high as $\eta_{I R-S T C}=3.5$ is achievable.

\section{B. Distributed Interleaver Design}

The generation of distributed random interleavers used in our IRSTC aided MSC should be carried out in an efficient manner, while at the same time maintaining their random nature. In this paper, we propose an interleaver, which we refer to as Structured Embedded (SE) interleaver. The SE interleavers are constructed from three hierarchical layers, namely from a system-specific base interleaver, a source-specific base interleaver and a so-called constituent interleaver set. These interleavers are then subjected to a position sorting operation, all of which are detailed below.

The system-specific base interleaver $\pi^{b}$ is a randomly generated interleaver of length- $Q$. Additionally, each AS has a distinct sourcespecific base interleaver $\pi_{k}^{b}, k \in[1, K]$ having the same length- $Q$ as the system-specific base interleaver $\pi^{b}$. The $(k+1)$ st source-specific base interleaver used in the $(k+1)$ st TDD slot is an interleaved version of the $k$ th source-specific base interleaver used in the TDD slot $k$, which was rearranged by the system-specific base interleaver $\pi^{b}$, as follows: $\pi_{k+1}^{b}=\pi^{b}\left(\pi_{k}^{b}\right)$ and $\pi_{1}^{b}=\pi^{b}$.

The constituent interleaver set of AS $k$ is represented by $U$ number/level of length- $Q$ interleavers, which is formulated as $\pi_{k}^{c}=$ $\left\{\pi^{1}, \pi^{2}, \ldots, \pi^{U}\right\}$. Each element $\pi^{u} \in \pi_{k}^{c}, u \in[1, U]$ of the constituent interleaver set is a distinct length- $Q$ interleaver, having the same length as the system-specific base interleaver $\pi^{b}$. The $(u+1)$ st constituent interleaver is an interleaved version of the $u$ th constituent interleaver, which was rearranged by the source-specific base interleaver $\pi_{k}^{b}$, according to $\pi^{u+1}=\pi_{k}^{b}\left(\pi^{u}\right)$ and $\pi^{1}=\pi_{k}^{b}$.

Finally, the $U$ number of length- $Q$ interleavers are concatenated to form a unique length- $U Q$ interleaver. This is carried out by the constituent interleaver set position sorting operation, as defined by the position mapping function $f$, which maps the index $q^{u} \in[1, Q]$ within all the $U$ number of length- $Q$ constituent interleavers $\pi^{u} \in$ $\pi_{k}^{c}, u \in[1, U]$ into a single source-specific interleaver $\pi_{k}=f\left(\pi_{k}^{c}\right)$. From a different perspective, this implies unambiguously mapping the $U Q$ number of input bit positions to the interleaved positions $q \in$ $[1, U Q]$. More specifically, the index $q^{u} \in[1, Q]$ within any of the $U$ number of length- $Q$ constituent interleavers $\pi^{u}, u \in[1, U]$ is mapped to $q=\left(q^{u}-1\right) U+u$. These indexes are stored in an intermediate interleaver $\hat{\pi}_{k}$ and the resultant source-specific interleaver $\pi_{k}$ obeys $\pi_{k}=\hat{\pi}_{k}\left(\hat{\pi}_{k}\right)$. 


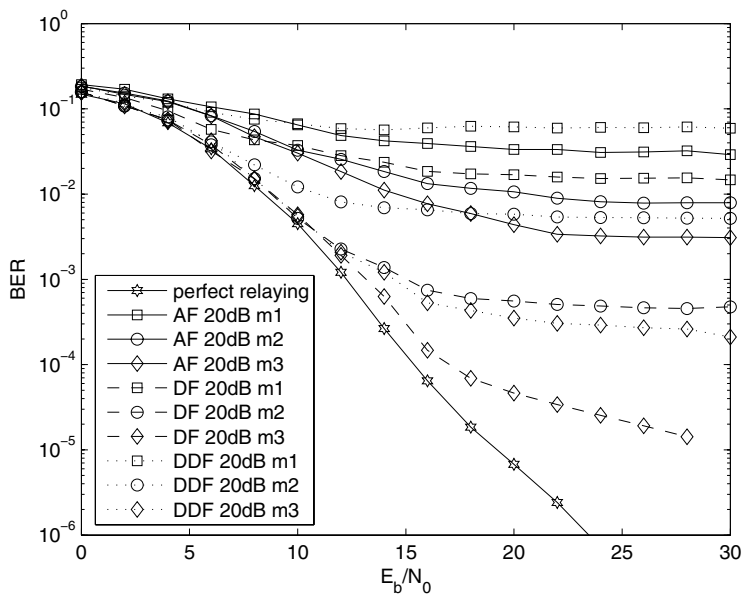

Fig. 2. Comparison of AF, DF and DDF relaying techniques employed in the 4-layer IR-STC aided MSC scheme, when the inter-source channel SNR was $\gamma_{s, s}=20 d B$.

\section{Simulation Results}

In this section, we investigate the performance of our IR-STC aided MSC employing different relaying techniques and stipulating different assumptions concerning $h_{s, s}$ by varying the Nakagami-m fading parameters. Both $h_{s, s}$ and $h_{s, d}$ are assumed to be quasi-static, i.e. constant in every 1024 information symbol block, but they are independently faded between the consecutive blocks. The value of $h_{s, d}$ is assumed to be perfectly known at the destination, while the knowledge of $h_{s, s}$ is either explicitly required or dispensed with at both the CSs and the destination, depending on the specific relaying technique employed. In all of our simulations, perfect relaying implies that all $K$ ASs' information bits are perfectly known at all $N$ CSs. Our simulation parameters are listed in Table. I.

Fig. 2 characterises three different relaying techniques, namely AF, DF and DDF employed in our IR-STC aided MSC scheme, when the inter-source channel SNR was $\gamma_{s, s}=20 \mathrm{~dB}$. As expected, the higher the value of the Nakagami parameter $m$, the less hostile the channel fading encountered, which results in an improved BER performance for all the three relaying techniques. For all three $m$ values considered, DF leads to the best BER performance, while the performance attained by DDF is better than that of AF, except for $m=1$. The worst performance of AF relaying is mainly a consequence of its noise enhancement. To elaborate a little further, the inferior performance of DDF compared to that of DF is a direct consequence of its doubled noise variance, when non-coherent detection was employed. For $m=1$, the effect of noise enhancement imposed by AF relaying is less severe than that of the doubled noise variance of non-coherent detection encountered by DDF, as evidenced by the results of Fig. 2 .

Similar conclusions can also be inferred from Fig. 3, which characterises the three different relaying techniques, when the intersource channel SNR was $\gamma_{s, s}=30 d B$. The inferior performance imposed by the doubled noise variance of non-coherent detection in DDF compared to DF was less obvious for $m>1$, since at $\gamma_{s, s}=30 \mathrm{~dB}$ both DF and DDF become capable of near-error-free detection, while detection errors persist for $m=1$ in both techniques. Likewise, it can be seen in Fig. 3 that the discrepancy between $\mathrm{AF}$ and DF becomes small because of the less pronounced noise enhancement, when we have benign fading. However, the difference of AF and DF remains relatively high in Fig. 4, which characterises all the three relaying techniques at an inter-source channel SNR $\gamma_{s, s}=30 \mathrm{~d} B$, when supporting $L=7$ layers per CS. In this case, the

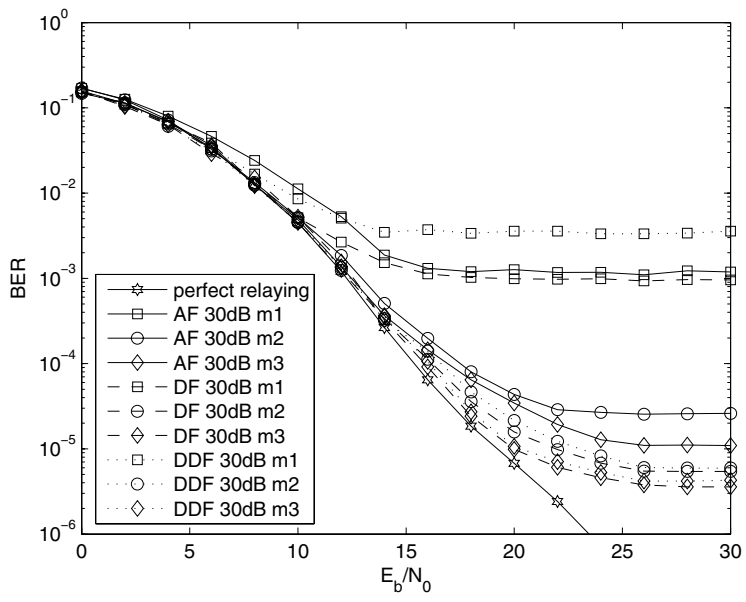

Fig. 3. Comparison of AF, DF and DDF relaying techniques employed in the 4-layer IR-STC aided MSC scheme, when the inter-source channel SNR was $\gamma_{s, s}=30 \mathrm{~dB}$.

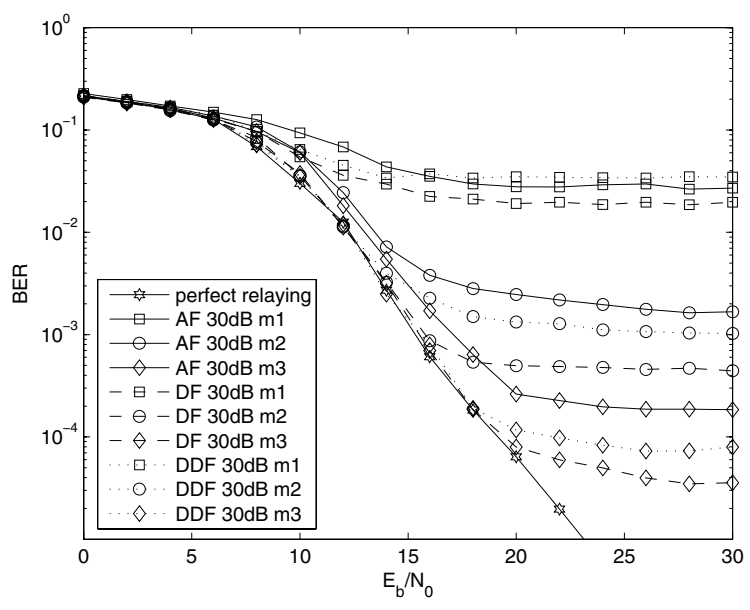

Fig. 4. Comparison of AF, DF and DDF relaying techniques employed in the 7-layer IR-STC aided MSC scheme, when the inter-source channel SNR was $\gamma_{s, s}=30 \mathrm{~dB}$.

effect of noise enhancement is further aggravated by superimposing more layers for the sake of achieving a high throughput, although each noise contribution itself may be relatively modest.

Fig. 5 compares two non-regenerative relaying techniques, namely AF and SDF employed in our IR-STC aided MSC scheme, when the inter-source channel SNR was $\gamma_{s, s}=30 d B$. It can be seen that there is only an insignificant difference between these two techniques ${ }^{4}$. However, as discussed in Section II, when the AF technique is employed, the knowledge of $h_{s, s}$ is required at the destination. By contrast, only the knowledge of $\left|h_{s, s}\right|$ is required at the destination, when SDF is employed. It can be seen in Fig. 5 that when a carrier phase error of $\phi=\pi / 16$ is imposed on $h_{s, s}$ at the destination, a significantly reduced BER performance is observed. This implies that $\mathrm{SDF}$ is a better relaying technique compared to $\mathrm{AF}$, provided that the CSs are capable of acquiring accurate knowledge of $h_{s, s}$. However, having the knowledge of $\left|h_{s, s}\right|$ at the CS is sufficient for ensuring reliable operation of the $\mathrm{AF}$ technique.

\footnotetext{
${ }^{4}$ In uncoded or repetition coded systems, SDF essentially becomes an AF technique, which is inferior to the DF technique. When a serial concatenated outer channel code is employed, SDF becomes capable of enabling soft channel decoding and the corresponding extrinsic information $\mathcal{L}$ becomes more reliable. This results in a higher mutual information $I\left(x_{k}^{(1)}\right)$, which is equivalent to having a reduced noise variance. Therefore, the achievable performance is expected to be better than that of DF.
} 


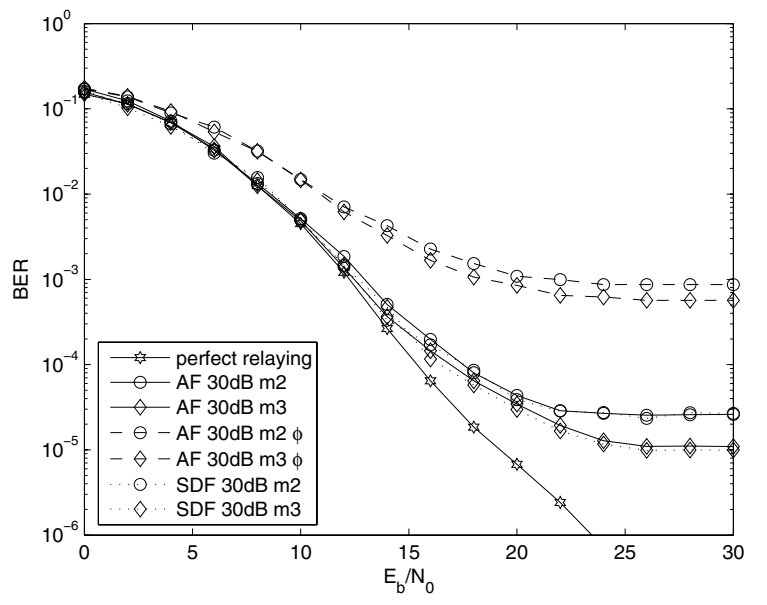

Fig. 5. Comparison of AF, SDF and AF subject to carrier phase error at the destination employed in the 4-layer IR-STC aided MSC scheme, when the inter-source channel SNR was $\gamma_{s, s}=30 d B$.

All of our previous investigations were based on having a fixed inter-source channel SNR. To expound further, Fig 6 shows three different relaying techniques, namely AF, DF and DDF employed in our IR-STC aided MSC, when the channel $h_{s, s}$ experiences different Nakagami-m fading and assuming a consistently higher SNR value than that associated with $h_{s, d}$, i.e. we have $\Delta=\gamma_{s, s} / \gamma_{s, d}>0 d B$. It can be seen in Fig. 6 that for $m=2$ DF performs consistently better than the other two techniques and approaches the perfect relaying performance, namely that of the system, which regenerates the source information without decision errors. Surprisingly, DDF also performs consistently better than the AF technique. However, when severe Rayleigh fading is encountered, i.e. we have $m=1$, AF has the best performance at high SNR, where the effect of noise enhancement is negligible. By contrast, the performance of both DF and DDF are unacceptable, owing to the effects of Rayleigh fading. Therefore, ideally the specific relaying technique used should be determined according to the specific Nakagami-m fading values encountered. This suggests switching amongst the different relaying modes.

Remarks: We may now conclude that when the SNR of the $h_{s, s}$ channel is better than that of $h_{s, d}$, DF is the best relaying strategy in the presence of benign fading. When a sufficiently high-SNR benign faded $h_{s, s}$-channel is experienced, close-to-perfect relaying performance is attainable. The $\mathrm{AF}$ technique is only preferable at high SNRs when severe fading is encountered. DDF performs consistently worse than DF due to the doubled noise variance of non-coherent detection. Surprisingly, when the fading is benign, non-coherent DDF without the cost of estimating all inter-source channel knowledges outperforms the coherent detected AF technique. Therefore, a preselection of the CSs benefiting from a high-SNR $h_{s, s}$ channel-which typically also have high Nakagami-m values-is important in MSC.

\section{CONCLUSION}

In this paper, we analyzed the achievable performance of our IRSTC aided MSC employing various relaying techniques. The slot utilisation efficiency of our MSC was characterised and a novel SE random interleaver generation method was proposed. Moreover, three different relaying techniques were compared. Our IR-STC design is capable of achieving a high throughput, while maintaining a low BER with the aid of decentralized cooperation. The non-orthogonal nature of our IR-STC facilitates near-capacity designs. These properties render our IR-STC design eminently applicable for employment in interference-limited high-user-density ad hoc networks.

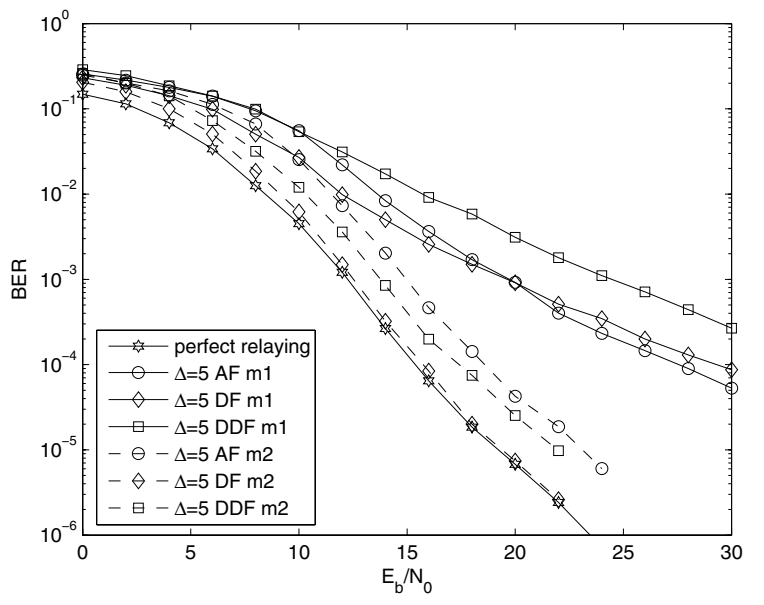

Fig. 6. Comparison of AF, DF and DDF relaying techniques employed in the 4-layer IR-STC aided MSC scheme having inter-source channel SNR to source-destination channel SNR ratio $\Delta=5$, when $h_{s, s}$ is subjected to Nakagami fading associated with $m=1$ and $m=2$.

\section{REFERENCES}

[1] A. Sendonaris, E. Erkip, and B. Aazhang, "User cooperation diversity. Part I and II," IEEE Transactions on Communications, vol. 51, pp. 19271948, Nov. 2003.

[2] R. Pabst, B. Walke, D. Schultz, P. Herhold, H. Yanikomeroglu, S. Mukherjee, H. Viswanathan, M. Lott, W. Zirwas, M. Dohler, H. Aghvami, D. Falconer, and G. Fettweis, "Relay-based deployment concepts for wireless and mobile broadband radio," IEEE Communications Magazine, vol. 42, pp. 80-89, Sept. 2004.

[3] A. Ozgur, O. Leveque, and D. Tse, "Hierarchical cooperation achieves optimal capacity scaling in ad hoc networks," IEEE Transactions on Information Theory, vol. 53, pp. 3549-3572, Oct. 2007.

[4] J. Laneman, D. Tse, and G. Wornell, "Cooperative diversity in wireless networks: Efficient protocols and outage behavior," IEEE Transactions on Information Theory, vol. 50, pp. 3062-3080, Dec. 2004.

[5] G. Kramer, M. Gastpar, and P. Gupta, "Cooperative strategies and capacity theorems for relay networks," IEEE Transactions on Information Theory, vol. 51, pp. 3037-3063, Sept. 2005.

[6] K. Azarian, H. E. Gamal, and P. Schniter, "On the achievable diversity multiplexing tradeoff in half-duplex cooperative channels," IEEE Transactions on Information Theory, vol. 51, pp. 4152-4172, Dec. 2005.

[7] O. Shalvi, "Multiple source cooperation diversity," IEEE Communications Letters, vol. 8, pp. 712-714, Dec. 2004.

[8] A. Ribeiro, R. Wang, and G. Giannakis, "Multi-source cooperation with full-diversity spectral-efficiency and controllable-complexity," IEEE Journal on Selected Areas in Communications, vol. 25, pp. 415-425, Feb. 2007.

[9] K. Wu and L. Ping, "Multilayer turbo space-time codes," IEEE Transactions on Communications, vol. 9, pp. 55-57, Jan. 2005.

[10] R. Zhang and L. Hanzo, "Space-time coding for highthroughput interleave division multiplexing aided multi-source cooperation," IEE Electronics Letters, Available online from http://eprints.ecs.soton.ac.uk/15247/.

[11] L. Hanzo, T. Liew, and B. Yeap, Turbo Coding, Turbo Equalisation and Space-Time Coding for Transmission over Fading Channels. WileyIEEE Press, 2002.

[12] X. Ma and L. Ping, "Coded modulation using superimposed binary codes," IEEE Transactions on Information Theory, vol. 50, pp. 33313343, Dec. 2004.

[13] E. Larsson and B. Vojcic, "Cooperative transmit diversity based on superposition modulation," IEEE Communications Letters, vol. 9, pp. 778-780, Sept. 2005.

[14] R. Steele and L. Hanzo, Mobile Radio Communications, 2nd ed. IEEE Press - John Wiley, 1999.

[15] S. ten Brink, "Convergence behavior of iteratively decoded parallel concatenated codes," IEEE Transactions on Communications, vol. 49, pp. 1727-1737, Oct. 2001.

[16] B. Mergen and A. Scaglione, "Randomized space-time coding for distributed cooperative communication," IEEE Transactions on Signal Processing, vol. 55, pp. 5003-5017, Oct. 2007. 